\title{
Results of a Pilot Study and a Proposal to Build a High Current Pulsed Nanosecond Low Energy Si Ion Beam for the Detection of Trace Amounts of Heavy Impurities in Silicon.
}

\author{
BNL Informal Report \#62332
}

\author{
F.M. Jacobsen, M.J. Zarcone, M. Cholewa*, D. Steski, K. Smith, \\ P. Thieberger, K.G. Lynn and J. Throwe \\ Brookhaven National Laboratory \\ Upton, NY 11973
}

RECEIVED

\begin{abstract}
Next generations of Very Large Scale Integrated circuits will require impurity contamination below $10^{8}$ atoms $/ \mathrm{cm}^{2}$. To detect such small quantities at or near the surface, new techniques have to be developed. We propose to build a high current nanosecond pulsed Si ion beam which can detect such small quantities of heavy impurities with a high mass resolution. A pilot study shows that our approach can be used to detect impurities in silicon below the $10^{7}$ atoms $/ \mathrm{cm}^{2}$ level.

\section{DISCLAIMER}

This report was prepared as an account of work sponsored by an agency of the United States Government. Neither the United States Government nor any agency thereof, nor any of their employees, makes any warranty, express or implied, or assumes any legal liability or responsibility for the accuracy, completeness, or usefulness of any information, apparatus, product, or process disclosed, or represents that its use would not infringe privately owned rights. Reference herein to any specific commercial product, process, or service by trade name, trademark, manufacturer, or otherwise does not necessarily constitute or imply its endorsement, recommendation, or favoring by the United States Government or any agency thereof. The views and opinions of authors expressed herein do not necessarily state or reflect those of the United States Government or any agency thereof.
\end{abstract}

JANO 31995

OSTI

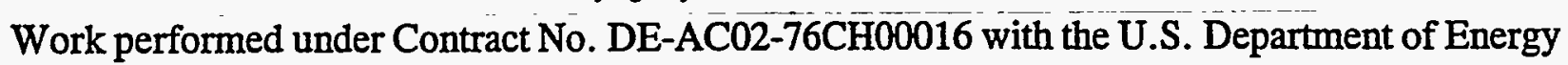

*Micro Analytical Research Centre (MARC), School of Physics, The University of Melbourne, Parkville, Vic. 3052, Australia

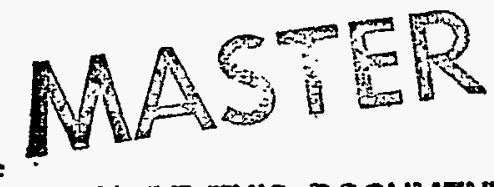



1. Introduction

At the turn of the century, the next generations of Very Large Scale Integrated circuits will require impurity contamination below $10^{8}$ atoms $/ \mathrm{cm}^{2}$ [1-2]. To detect such small quantities at or near the surface, new techniques are being developed. Of these, the Time Of Flight - Heavy Ion Backscattering Spectroscopy, TOF-HIBS, developed by Sandia [3-6] seems the most promising. TOF-HIBS is based on the following relationship between the velocity of the incoming projectile, $v_{p}$, and that of the backscattered projectile, $v_{s}$,

$$
v_{s}=\frac{M_{p}-M_{t}}{M_{p}+M_{t}} \cdot v_{p}
$$

where $M_{p}$ is the mass of the incoming beam and $M_{t}$ is the mass of the target atoms.

An important ingredient in the Sandia experimental setup is a thin carbon foil in the flight path of the detectable backscattered ions. The detection of the secondary electrons emitted from this foil supplies the stop signal (through a delay) whereas the detection of the backscattered ions gives the start signal to produce an inverted TOF spectrum. In addition, a careful selection of the thickness of the carbon foil will prevent ions backscattered off the silicon substrate atoms from hitting the ion detector (the Sandia group is using ion beams lighter ( $\mathrm{C}$ and $\mathrm{N}$ ) than that of 
the silicon substrate atoms (see Eq. 1)). The use of the carbon foil causes some energy straggling of the backscattered ions with the result of some degradation of time and therefore mass resolution of their spectrometer. As of 1994 , their sensitivity for $\mathrm{Au}$ on $\mathrm{Si}$ was $10^{9}$ atoms $/ \mathrm{cm}^{2}$ [3]. With the addition of more detectors a sensitivity for $\mathrm{Au}$ of $10^{8}$ atoms $/ \mathrm{cm}^{2}$ is anticipated by the Sandia group while for lighter elements the sensitivity is somewhat less $\left(5 \times 10^{9}\right.$ atoms $/ \mathrm{cm}^{2}$ for $\mathrm{Fe}$ ).

The use of the carbon foil is what allows the Sandia group to measure small amounts of heavy impurities in $\mathrm{Si}$. However, this carbon foil also represents the limitation of their approach. The mass resolution of the Sandia spectrometer is not adequate at this time but can be improved somewhat by increasing the flight path of the backscattered ions until the resolution becomes limited by energy straggling of the ions in the carbon foil. Improving the Time Of Flight resolution results in better mass resolution and, what is equally important, improves the sensitivity toward heavy impurities simply by improving the signal to noise ratio of the spectrometer.

Our approach is to use a pulsed ion beam which eliminates the need for producing secondary electrons to provide a start signal for the Time Of Flight measurements. Secondly, by using a Si ion beam, backscattering from the substrate is eliminated and, therefore, the carbon foil is no longer required: 
To complete this introduction, we mention that other techniques can be used to detect impurities at or near the surface of silicon. The most sensitive of these is probably $\mathrm{x}$-ray fluorescence which can detect $10^{10}$ atoms $/ \mathrm{cm}^{2}$ of elements such as $\mathrm{Fe}, \mathrm{Ni}$ and $\mathrm{Zn}$, whereas for other elements the sensitivity is limited to $10^{12}$ atoms $/ \mathrm{cm}^{2}$. Here, we define the detection limit to occur when the signal rate equals the background rate and this definition is used throughout this paper. It is of course possible to define the elemental sensitivity differently as done in ref. [7] whereby they obtain a detection limit for $\mathrm{Fe}, \mathrm{Ni}$ and $\mathrm{Zn}$ in the range $2.5-4.3 \times 10^{8}$ atoms $/ \mathrm{cm}^{2}$.

In Section 2, we discuss the results of our preliminary study of using a pulsed Si beam to detect heavy impurities in silicon. Section 3 gives details of our proposed high current pulsed nanosecond low energy Si beam. A summary and conclusion are given in Section 4.

2. Results of a pilot study using a Si beam to detect trace amounts of impurities in silicon.

In a feasibility study, an existing simple non-UHV pulsed $\mathrm{Si}^{-}$beam line was used to measure the concentration of $\mathrm{Cu}$ and $\mathrm{Au}$ on the surfaces of graphite and silicon targets. The $30 \mathrm{keV} \mathrm{Si}$ ions were chopped into 200 nsec. pulses then entered the target chamber through a $1 \mathrm{~mm}$ diameter aperture in a $15 \mathrm{~cm}$ diameter Channel Electron Multiplier Array, CEMA, detector. The CEMA detects the backscattered Si ions/atoms from the target to deliver start signals for a Time to Amplitude Convertor. The stop signals to the TAC are derived from machine pulses. This arrangement results in an inverted TOF spectrum of the backscattered Si ions/atoms. The 
distance between the target and the CEMA detector is $48 \mathrm{~cm}$ resulting in typical flight times of the backscattered $\mathrm{Si}$ in the $\mu \mathrm{sec}$ range.

Fig. 1 shows a TOF spectrum using $30 \mathrm{keV} \mathrm{Si}$ for $1.3 \times 10^{13}$ atoms $/ \mathrm{cm}^{2}$ of $\mathrm{Au}$ on a silicon surface. By taking the result of Fig. 1 as is, we can conclude that the sensitivity of this preliminary feasibility test is on the order of $10^{11}$ atoms $/ \mathrm{cm}^{2}$ of $\mathrm{Au}$ on a Si surface. By improving the time resolution over the present 200 nsec. to about 1 nsec. the sensitivity becomes $10^{9}$ atoms $/ \mathrm{cm}^{2}$ for $\mathrm{Au}$ on a silicon surface, a number which is comparable to the Sandia results.

However, we can do much better than that. The TOF spectrum in Fig. 1 was obtained by considerably reducing the gain of the CEMA. This point is illustrated in Fig. 2 which compares the result of Fig. 1 where the voltage across the CEMA was $V_{\text {CEMA }}=1750$ volts to a TOF spectrum measured for the same target with $\mathrm{V}_{\text {CEMA }}=2100$ volts. As observed, by increasing $\mathrm{V}_{\text {CEMA }}$ the Au peak area counts increase from $\sim 200 / \mathrm{nC}$ to $\sim 3600 / \mathrm{nC}$ corresponding to a signal enhancement of 18 . Unfortunately, the background rate mainly associated with surface hydrogen increases out of proportion causing the signal to noise ratio to drop about two orders of magnitude. The hydrogen background is a result of a double scattering process in which an incoming $\mathrm{Si}^{-}$hits a surface $\mathrm{H}$ which then backscatters off a substrate $\mathrm{Si}$ atom. Note that the $\mathrm{H}$ background is also observed in Fig. 1 as a small structural background observed at the wings of the Au peak. The additional uniform background in Fig. 1 is caused by neutralization of some of the $\mathrm{Si}^{-}$beam by rest gases in the beam pipes. This background is easily removed by improving the beam pipe vacuum. 


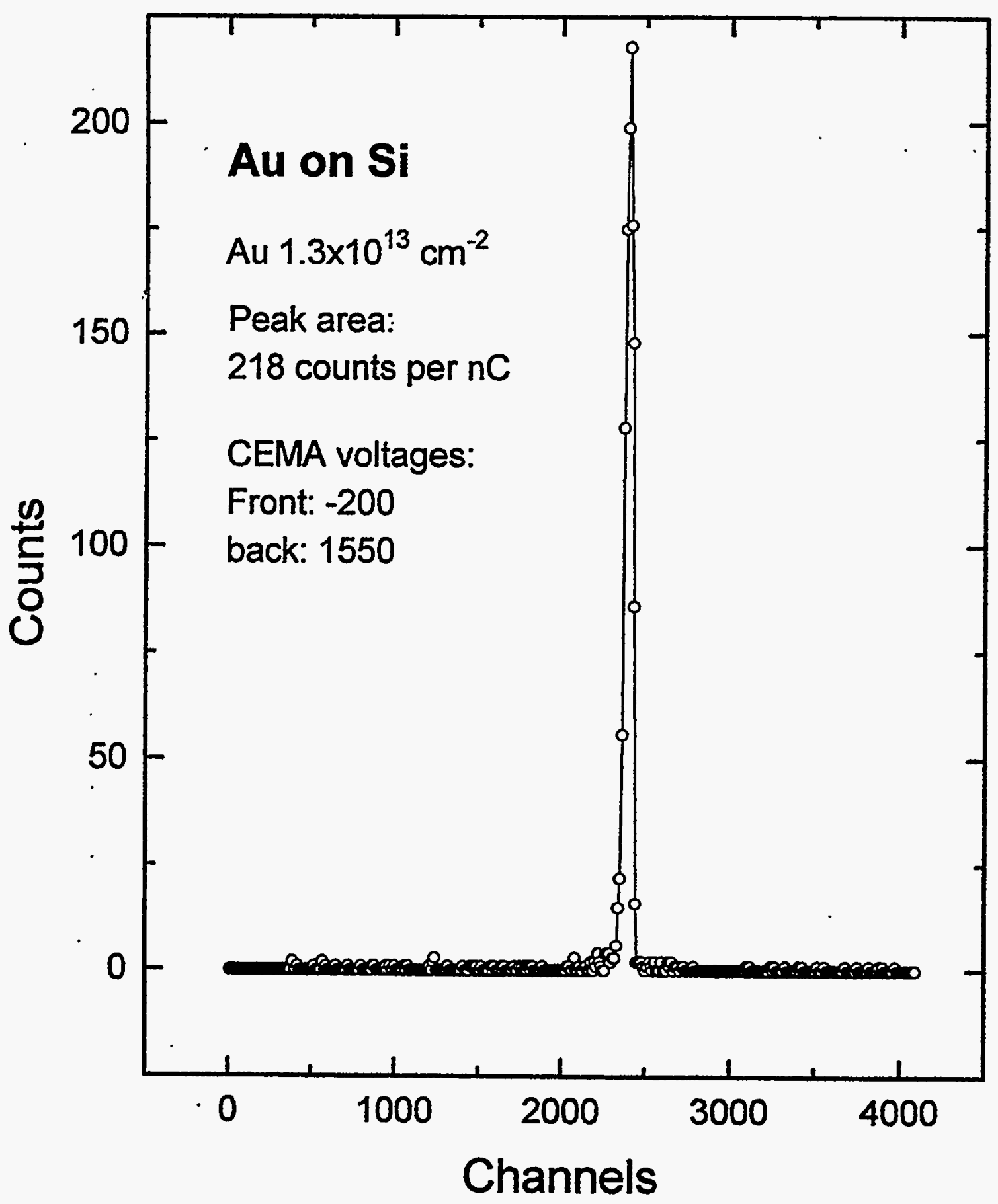

Figure 1. : Time of Flight Spectrum for Au on Si. 


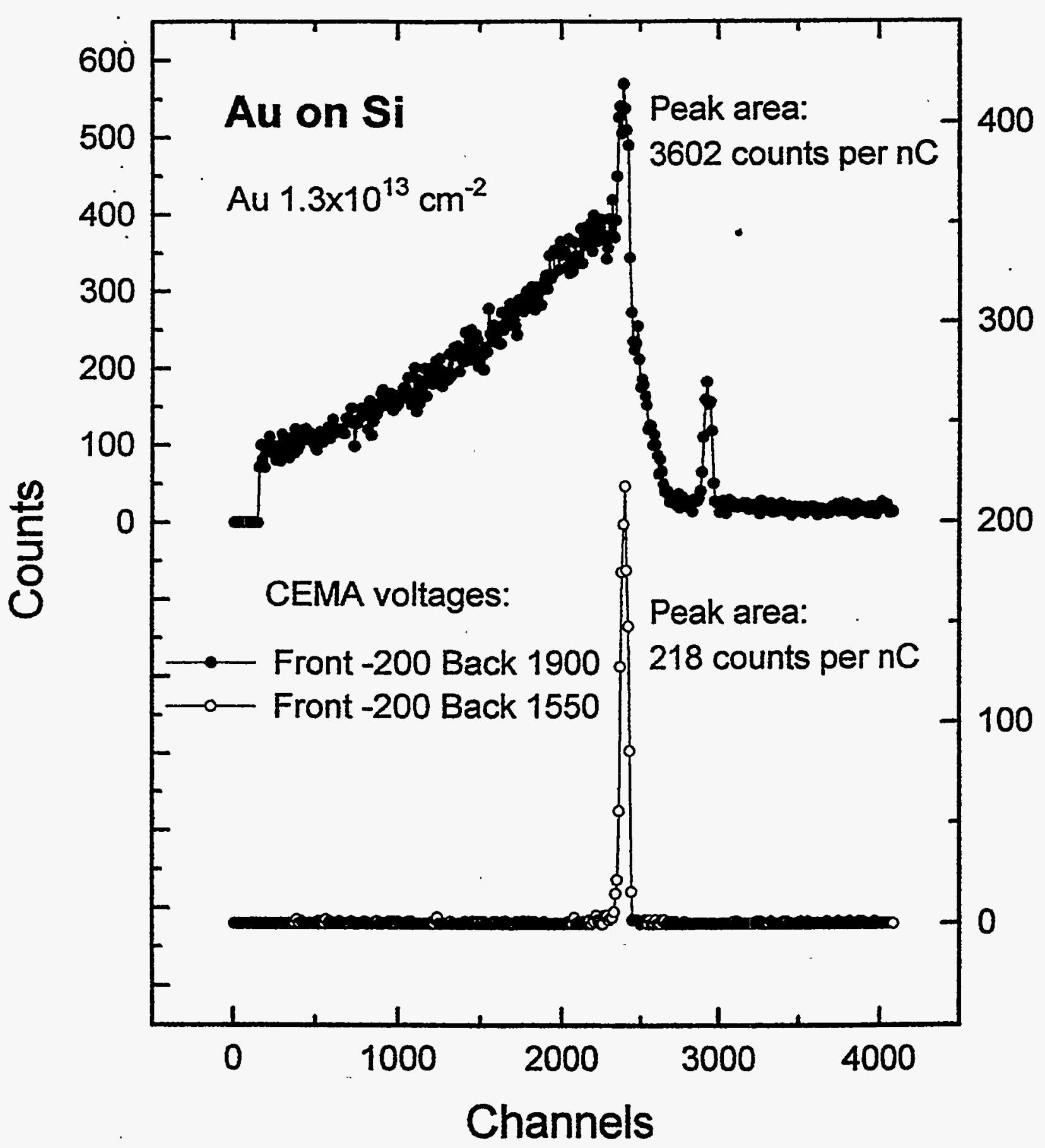

Figure 2. : TOF Spectrum for Au on Si. (Top) $V_{\text {CEMA }}=2100$ Volts, (bottom) $\mathrm{V}_{\text {CEMA }}=1750$ Volts 
The reason for the markedly different results of the two spectra in Fig. 2 is that the charge state of the backscattered $\mathrm{Si}$ off $\mathrm{Au}$ is +1 , whereas the hydrogen contribution stems mainly from backscattered neutral hydrogen. The charge state of the backscattered Si was determined by measuring the shifts of the TOF peak versus target bias. The detection efficiency of the CEMA at low bias is considerably higher for the charged particles as compared to the neutral ones at the impact velocities in question. A simple way to eliminate the neutral hydrogen contribution is to position a thin foil of either carbon or alumina in the front of the CEMA detector and use this foil to convert the ions into electrons by accelerating the backscattered $\mathrm{Si}$ ions into this ion electron convertor. Note, that this foil will be positioned at the end of the flight path of the backscattered ions and therefore it does not result in degradation of mass resolution.

To be conservative, it does seem reasonable to expect a sensitivity for Au at or near the surface of silicon to approach the $10^{8}-10^{7}$ atoms $/ \mathrm{cm}^{2}$ level when a low energy pulsed Si beam is applied.

At the low energy used here the backscattering cross - section is of atomic dimension and therefore we will expect the sensitivity toward other heavy impurities in silicon to be comparable to that of Au providing the Si backscatters in a charge state of +1 .

That this is not always the case is illustrated in Fig. 3a which shows a TOF spectrum using $30 \mathrm{keV} \mathrm{Si}$ for high coverage of $\mathrm{Au}$ and $\mathrm{Cu}$ on graphite. This spectrum was taken with the high gain of the CEMA. The peak due to the $\mathrm{Cu}$ is barely seen, the reason is that the $\mathrm{Si}$ backscatters 


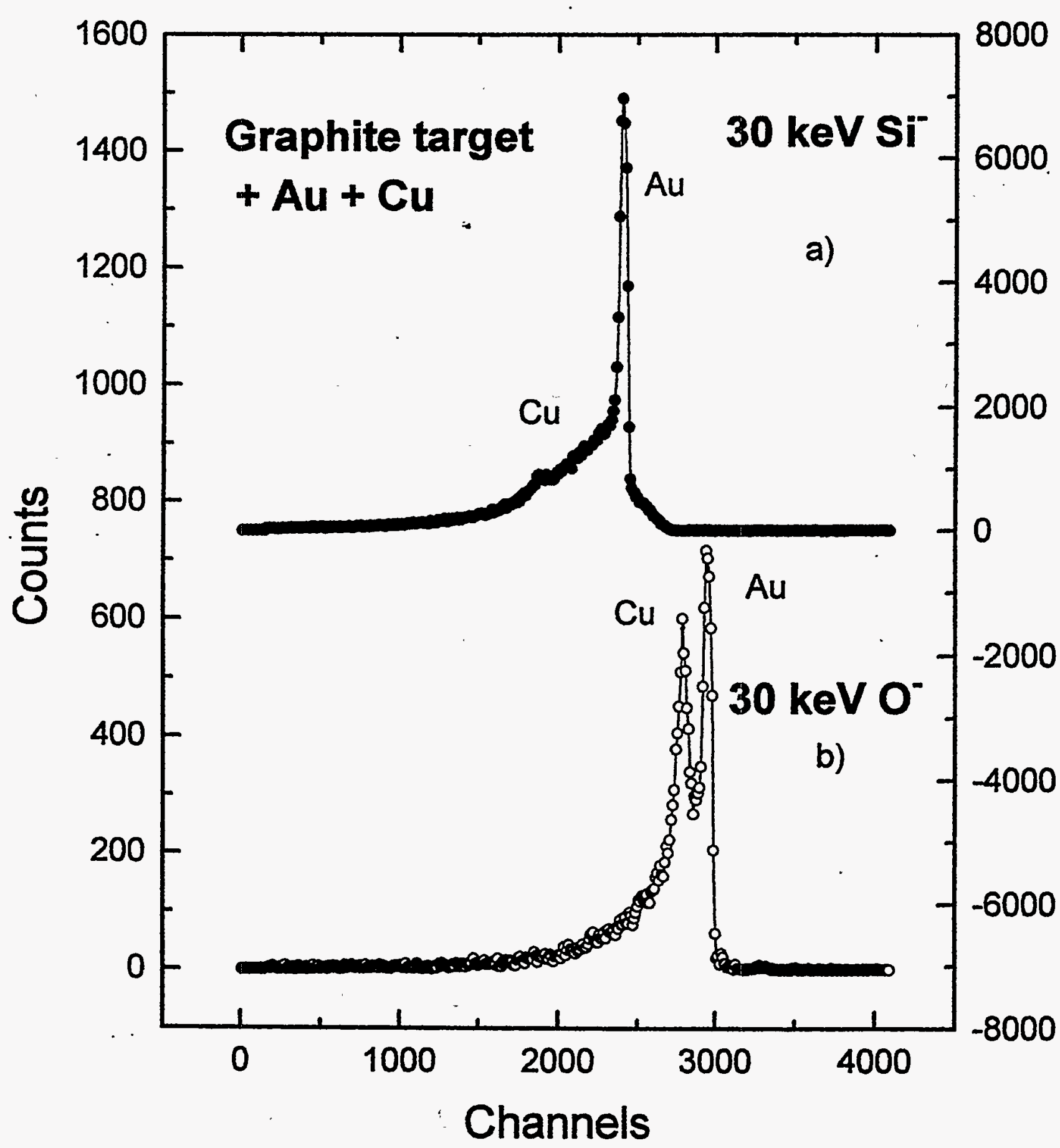

Figure 3. : TOF Spectra for $\mathrm{Au}$ and $\mathrm{Cu}$ on Graphite. a.) using a $30 \mathrm{kV}$ Si Beam b.) using a $30 \mathrm{kV} \mathrm{O}^{-}$Beam. 
off $\mathrm{Cu}$ in a neutral charge state. By changing to a $30 \mathrm{keV} \mathrm{O}$ beam, the backscattered signals due to $\mathrm{Cu}$ and $\mathrm{Au}$ become comparable (see Fig. $3 \mathrm{~b}$ ).

At the low impact velocity in question direct ionization is not possible and a collision should be viewed as to proceed through the formation of a transient molecular excited species formed by the projectile and the target atom [8-11]. The outcome of such a slow (adiabatic) collision depends on the details of the potential curves versus internuclear distance for the initial state and the various final states and if the initial state potential curve happens to come sufficiently close to a final state curve having the projectile in a charge state +1 then auto-ionization or charge transfer may occur. It is always possible to ensure that a sufficient fraction of the backscattered beam off any heavy atom is in a charge of +1 , either by changing the impact energy or by changing the projectile as illustrated in Fig. 3 . Of course a much simpler solution is to use a positively charged Si beam. In this preliminary study, it was not possible to test the latter approach.

3. A description of the proposed ion beam.

Our goal is to build a pulsed low energy ion beam which can generate positive and negative $\mathrm{Si}$ ions. Ignoring the pulsing feature, the ion beam is generated in the following way. A Cs source delivers $\mathrm{Cs}^{+}$ions which are accelerated to $5-10 \mathrm{keV}$ before they are focussed onto the primary ion source Si target. Both positive and negative Si ions are sputtered off the ion source target. In the pilot experiment, the $\mathrm{Cs}^{+}$source was positioned on the $\mathrm{Si}$ ion beam axis and 
therefore it was possible to extract only negative $\mathrm{Si}$ ions. By having the $\mathrm{Cs}^{+}$beam line at a $45^{\circ}$ angle to the primary beam line, positive ions can be extracted as well.

We have two options for pulsing the ion beam. The first one consists of chopping the DC beam into short time pulses of ions using a $2 \mathrm{MHz}$ beam sweeper operated at $2 \mathrm{kV}$ followed by a beam deflector. In the initial stage of this project which will be concerned with the optimization of the ion - electron convertor and comparing the energy dependence of the sensitivity of negative and positive Si ions toward heavy atom contamination of silicon, an improved version of this type of pulsing system with a time resolution of $10 \mathrm{nsec}$. will be used. By making this choice, we have taken the practical point of view that during the solving of these problems it makes sense to use a well known beam pulsing system.

Later, however, we will study a more efficient way of forming short time bunches of Si ions by bunching the Cs beam using a harmonic buncher before it hits the primary ion source target. This method ensures, that for practical purpose, no loss in current occurs as compared to a DC mode of operation.

Fig. 4 shows a sketch of the proposed ion beam. The ions generated (by the bunched $\mathrm{Cs}^{+}$ beam) at the ion source target are accelerated, focussed and transported to a $10 \mathrm{kV}$ accelerator whereafter the 1st. einzel lens focuses the beam onto the object slits of a $90^{\circ}$ analyzer magnet. When the beam exits the magnet's image slits it is further focussed by the second einzel lens, 


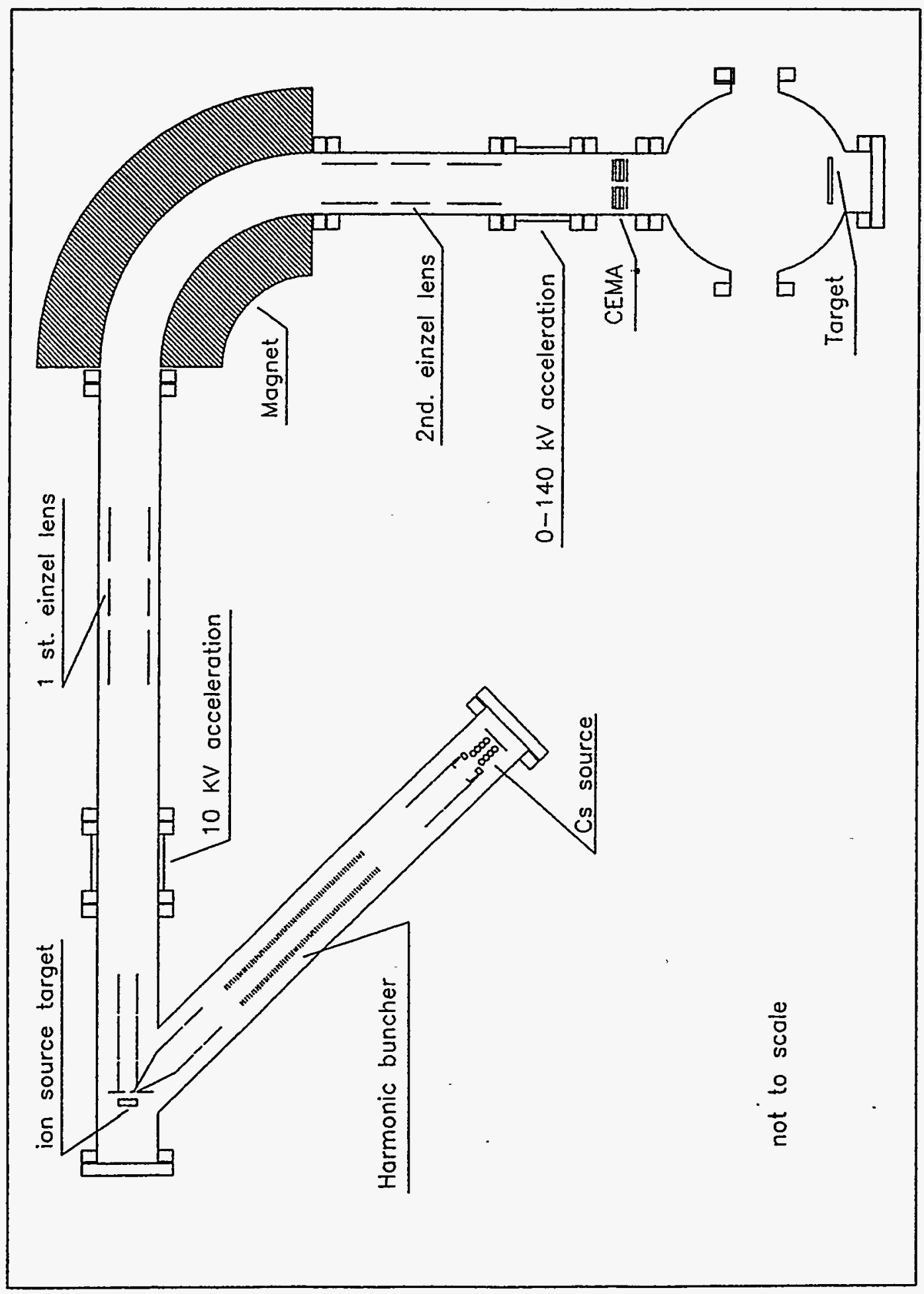

Figure 4. : Sketch of Proposed Beam Line. 
accelerated to final energy before it enters the target chamber through an aperture in a CEMA detector, and then hits the target. The CEMA detects the backscattered Si ions to supply start signals which, when combined with stop signals derived from the buncher potential, result in an inverted TOF spectrum.

The simple and yet remarkable property of a quadratic potential is that if an ion is started in this potential with zero velocity it will arrive at the end (or bottom) of this potential at a time which is independent of its initial position. The mechanical equivalent is the pendulum. What is lesser known is that if the ions possess an initial velocity a time focussing action will also take place, however, in this case it occurs at some distance past the quadratic potential. It is clear that any initial velocity of the ions will have the greatest effect the closer the start position is to the end of the buncher. Take two ions with the same initial velocity of which one (a) starts a quarter into the buncher and another (b) that is half the way in when the quadratic potential is pulsed on. For this case ion (b) will arrive at the end of the buncher before ion (a), however, the exit velocity of ion (a) is greater than that of ion (b) and therefore a time focussing point is formed at some distance past the end of the buncher for these particular ions.

In Fig. 5, the flight time versus distance past the end of the harmonic buncher is plotted for the case in which the buncher is half filled with Cs ions when the potential is pulsed on. The initial energy is assumed to be $100 \mathrm{eV}$ and the buncher voltage is $3 \mathrm{kV}$ whereas the length of the buncher is 1 meter. As observed, for those ions time focussing occurs at about $19 \mathrm{~cm}$ past the 


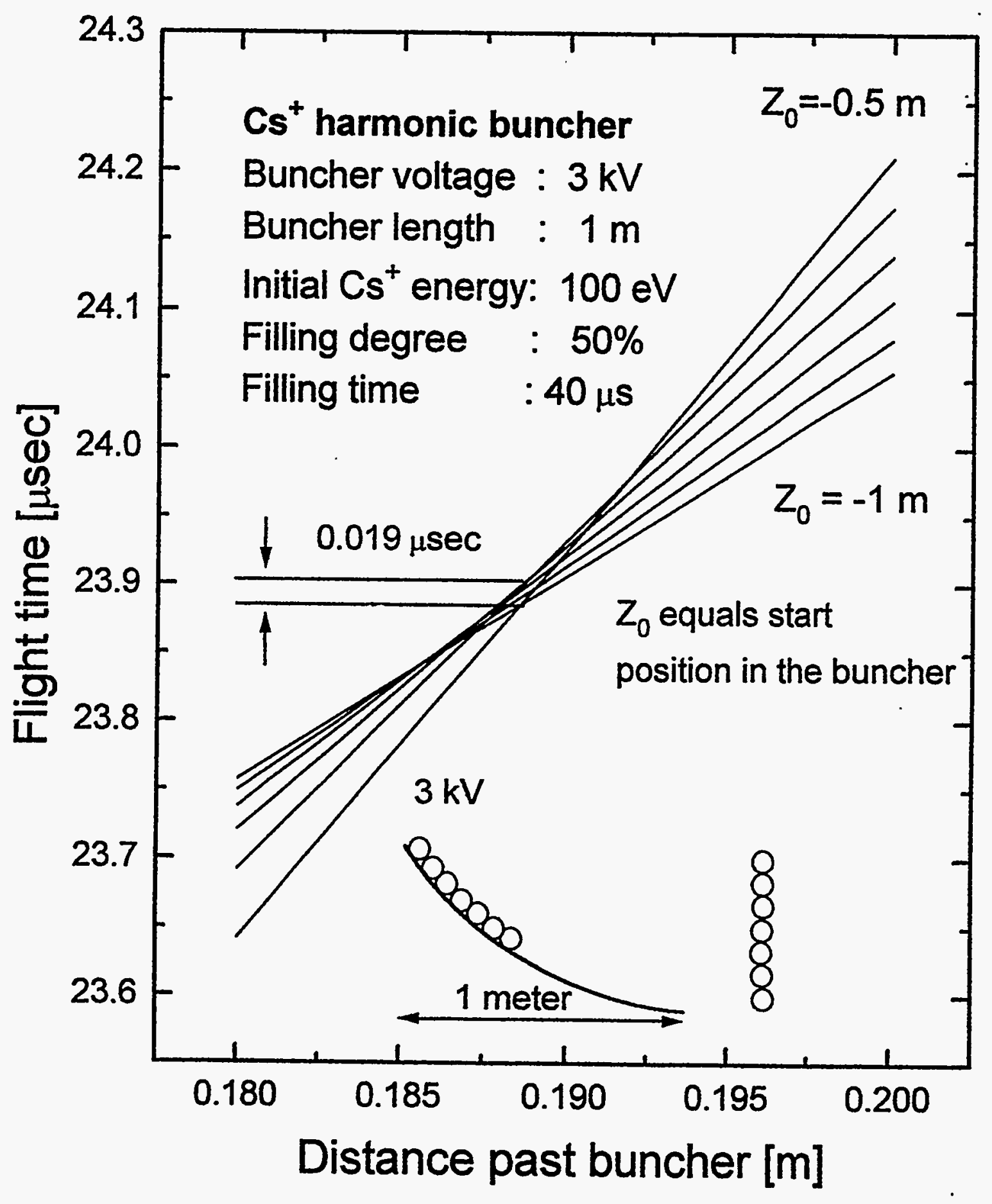

Figure 5. : Flight Time vs. Distance Past the End of the $\mathrm{Cs}^{+}$Buncher for Different Start Positions, $\mathbf{Z}_{0}$. 
buncher. In this example the time length, $\tau$, of the ion pulse is $19 \mathrm{nsec}$. However, as observed from Fig. 5, $\tau$ can be considerably reduced if the filling degree of the buncher is reduced. For example, by decreasing this quantity from $50 \%$ to $25 \%$ a time bunch of about 1 nsec. results.

During the transport of a $1 \mathrm{nsec}$. bunch of $\mathrm{Si}$ ions from the source region to the target chamber, a distance which is approximately 5 meters, one has to be concerned with time broadening of the $\mathrm{Si}$ ion bunch due to the following three factors 1 ) different trajectories along which the ions are transported, 2) slightly different velocities of the ions and 3) space charge effects in the ion bunch itself. Of these, probably, the space charge effect will be the limiting factor. However, at the $100 \mathrm{nA} \mathrm{DC}$ level the effect should be minimal. At this current level, the harmonic bunching approach will deliver two orders of magnitude more ions per second to the target chamber in 1 nsec. pulses as compared to a conventional beam sweeping - chopping system.

\section{Summary and conclusion remarks}

In a pilot study, we have demonstrated a sensitivity of $10^{11} \mathrm{Au}$ atoms $/ \mathrm{cm}^{2}$ on the surface of silicon using a simple pulsed low energy $\mathrm{Si}^{i}$ beam. The limiting factors were the wide time (200 nsec.) pulses of our beam, the vacuum in the beam pipes and the hydrogen contribution to the TOF signal. Simple methods to improve this sensitivity toward the $10^{7}$ atoms $/ \mathrm{cm}^{2}$ were discussed. Improving the time resolution to $1 \mathrm{nsec}$. results in a sensitivity level of $10^{9}$ atoms $/ \mathrm{cm}^{2}$ and if the hydrogen contribution can be completely eliminated then the ultimate sensitivity will be limited 
by sputtering effects of the silicon surface by our Si beam. A time resolution of $1 \mathrm{nsec}$. is good enough to make isotopic resolution possible for masses greater than the projectile mass.

For $\mathrm{Cu}$, on the other hand, the sensitivity of the Si- beam was low. This was due to that the $30 \mathrm{keV} \mathrm{Si}-$ beam backscatters off $\mathrm{Cu}$ in a neutral charge state which considerably reduces the detection efficiency of the CEMA. We argued that by changing the $\mathrm{Si}^{-}$energy or, as demonstrated, by using a different projectile ( $30 \mathrm{keV} \mathrm{O}^{-}$) it is possible to obtain the sensitivity for $\mathrm{Cu}$ and any other heavy atoms comparable to that for $\mathrm{Au}$. The simplest way to ensure this is to use a positively charged Si beam.

We emphasize that in order to achieve a sufficient sensitivity toward heavy impurities in silicon, it is not necessary to push all of the beam parameters in question to their limits. For example, by improving the time resolution from that of $200 \mathrm{nsec}$. used in the test experiment to 10 nsec. and by reducing the hydrogen contribution by a factor of 10 takes us to a sensitivity level below $10^{8}$ impurities $/ \mathrm{cm}^{2}$ if a positively charged $\mathrm{Si}$ beam is applied. The surface hydrogen contribution will be removed by using an ion - electron convertor combined with the CEMA to detect the backscattered Si ions.

On a final note, the proposed nanosecond pulsed low energy $(10-150 \mathrm{keV})$ ion beam can be viewed as a high performance general purpose surface tool which can be used within many areas of fundamental and applied science. In this respect, we mention that our ion sources can generate positive and negative ions from across the entire periodic table. 
Acknowledgements

The authors would like to thank Bob Sabatini for measuring the surface concentration of gold and copper on the target samples and Mario Manni for his engineering expertise.

\section{References}

1. A.C. Diebold, P. Maillot, M. Gordon, J. Baylis, J. Chacon, R. Witowski, H.F. Arlinghaus, J.A. Knapp and B.L. Doyle, J. Vac. Sci. Technol. 102945 (1992) .

2. S.J. Semiconductor Industry Association, San Jose, CA, Semiconductor Technology Workshop Working Group Reports (1993).

3. J.A. Knapp, J.C. Banks and B.L. Doyle, Nucl. Instrum. and Meth. B 8520 (1994).

4. J.A. Knapp and J.C. Banks, Nucl. Instrum. and Meth. B 79457 (1993).

5. J.A. Knapp and B.L. Doyle, Nucl. Instrum. and Meth B 45143 (1990).

6. B.L. Doyle, J.A. Knapp and D.L. Buller, Nuci. Instrum. and Meth. B 42295 (1989).

7. P. Pianetta, N. Takaura, S. Brennan, W. Tompkins, S.S. Laderman, A. Fischer-Colbrie, A. Shimazaki, K. Miyazaki, M. Madden, D.C. Wherry and J.B. Kortright, Rev. Sci. Instr. 661293 (1995).

8. S. Yu. Ovchinnikov and J.H. Macek, Nucl. Instr. and Meth. B 998 (1995).

9. H.S.W. Massey and H.B. Gilbody, "Electronic and Ionic Impact Phenomena" (The Clarendon Press, Oxford 1974).

10. U. Fano and W. Lichten, Phys. Rev. Lett. 14627 (1965).

11. M.J. Zarcone, "Ionization and Electron Emission of Heavy Ion - Atom Collisions: The Argon - Krypton Collision System" (Ph.D. Thesis, The University of Connecticut, 1989). 